University of Nebraska - Lincoln

DigitalCommons@University of Nebraska - Lincoln

2010

\title{
Communication with Aboveground Devices in Wireless Underground Sensor Networks: An Empirical Study
}

Agnelo R. Silva

University of Nebraska-Lincoln

Mehmet C. Vuran

University of Nebraska-Lincoln, mcvuran@cse.unl.edu

Follow this and additional works at: https://digitalcommons.unl.edu/cseconfwork

Part of the Computer Sciences Commons

Silva, Agnelo R. and Vuran, Mehmet C., "Communication with Aboveground Devices in Wireless Underground Sensor Networks: An Empirical Study" (2010). CSE Conference and Workshop Papers. 149. https://digitalcommons.unl.edu/cseconfwork/149

This Article is brought to you for free and open access by the Computer Science and Engineering, Department of at DigitalCommons@University of Nebraska - Lincoln. It has been accepted for inclusion in CSE Conference and Workshop Papers by an authorized administrator of DigitalCommons@University of Nebraska - Lincoln. 


\title{
Communication with Aboveground Devices in Wireless Underground Sensor Networks: An Empirical Study
}

\author{
Agnelo R. Silva and Mehmet C. Vuran \\ Department of Computer Science and Engineering \\ University of Nebraska-Lincoln, Lincoln, NE, 68588 \\ Email: \{asilva, mcvuran\}@cse.unl.edu
}

\begin{abstract}
Wireless Underground Sensor Networks (WUSNs) consist of wirelessly connected underground sensor nodes that communicate untethered through soil. WUSNs have the potential to impact a wide variety of novel applications including intelligent irrigation, environment monitoring, border patrol, and assisted navigation. Although its deployment is mainly based on underground sensor nodes, a WUSN still requires aboveground devices for data retrieval, management, and relay functionalities. Therefore, the characterization of the bi-directional communication between a buried node and an aboveground device is essential for the realization of WUSNs. In this work, empirical evaluations of underground-to-aboveground (UG2AG) and aboveground-to-underground (AG2UG) communication are presented. More specifically, testbed experiments have been conducted with commodity sensor motes in a real-life agricultural field. The results highlight the asymmetry between UG2AG and AG2UG communication for different burial depths. To combat the adverse effects of the change in wavelength in soil, an ultra wideband antenna scheme is deployed, which increases the communication range by more than $350 \%$ compared to the original antennas. The results also reveal that a $21 \%$ increase in the soil moisture decreases the communication range by more than $70 \%$. To the best of our knowledge, this is the first empirical study that highlights the effects of the antenna design, burial depth, and soil moisture on both UG2AG and AG2UG communication performance. These results have a significant impact on the development of multi-hop networking protocols for WUSNs.
\end{abstract}

Index Terms-Underground electromagnetic propagation; Wireless Underground Sensor Networks; Underground Communication

\section{INTRODUCTION}

Wireless Underground Sensor Networks (WUSNs) consist of wirelessly connected underground sensor nodes that communicate untethered through soil [2]. As a natural extension to the well-established wireless sensor network (WSN) paradigm [1], WUSNs are envisioned to provide underground monitoring capabilities in the fields of intelligent irrigation, environment monitoring, border patrol, and assisted navigation. Despite their potential advantages, however, the realization of WUSNs is challenging due to the significant and direct impact of soil characteristics and its dynamics on communication [3], [10]. More specifically, the changes in temperature, weather, soil moisture, soil composition, and depth directly impact the connectivity and communication success in underground settings.

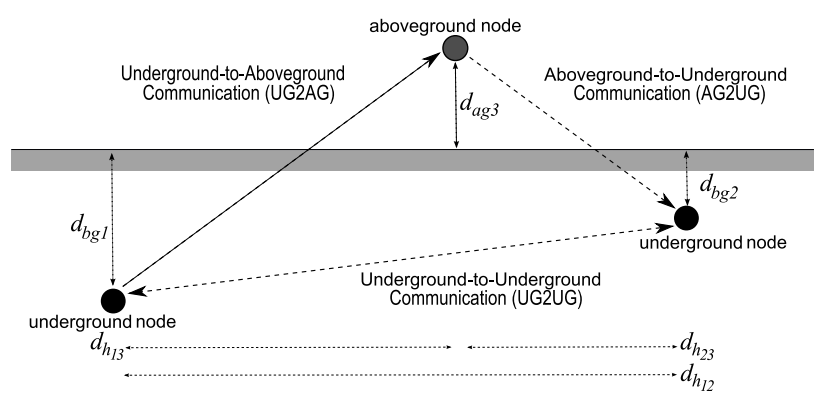

Fig. 1. Types of communication in WUSNs: UG2UG, UG2AG, and AG2UG.

Although its deployment is mainly based on underground sensor nodes, a WUSN still requires aboveground devices for data retrieval, management, and relay functionalities. Accordingly, three different communication links exist in WUSNs based on the locations of the transmitter and the receiver as shown in Fig.1:

- Underground-to-underground (UG2UG) Link: Both the sender and the receiver are buried underground and communicate through soil. This type of communication is employed for multi-hop information delivery.

- Underground-to-aboveground (UG2AG) Link: The sender is buried and the receiver is above the ground. Monitoring data is transferred to aboveground relays or sinks through these links.

- Aboveground-to-underground (AG2UG) Link: Aboveground sender node sends messages to underground nodes. This link is used for management information delivery to the underground sensors.

UG2AG and AG2UG links are required for several functionalities of WUSNs, such as network management and data retrieval. Thus, the characterization of the bi-directional communication between a buried node and an aboveground device is essential. Both the UG2AG and AG2UG links include underground propagation. Thus, the soil properties, such as soil moisture, directly impact the communication success. Moreover, the soil-air interface plays an important role in communication. Transmitted rays are reflected and attenuated at this interface, which significantly influences the channel quality. This influence also varies depending on the direction of the transmission (UG2AG or AG2UG). Furthermore, the wavelength of a radio frequency (RF) signal is significantly 
reduced when propagating through soil [11], [13]. The relation between the wavelength change and the design aspects of an underground antenna should be clearly identified.

To this end, in this work, the results of empirical evaluations for UG2AG and AG2UG communication are presented. More specifically, testbed experiments have been conducted with commodity sensor motes in a real-life agricultural field to characterize the factors on UG2AG and AG2UG links as mentioned above. We particularly consider agricultural applications of WUSNs, which usually require burial depths greater than $30 \mathrm{~cm}$ due to plowing and similar mechanical activities at the soil [10]. Accordingly, the majority of the experiments consider a $35-\mathrm{cm}$ burial depth.

The rest of this paper is organized as follows: In Section II, related work is discussed. In Section III, the methodology used in the experiments is described. Also, the ultra wideband antenna scheme used in the experiments is presented. The empirical results for the effects of the antenna type, burial depth, and soil moisture on the UG2AG/AG2UG communication performance are discussed in Section IV. Finally, a discussion of the results is provided in Section V.

\section{RELATED WORK}

Wireless underground communication has been investigated in many contexts recently. The concept of WUSNs and the challenges related to the underground wireless channel have been introduced in [2]. In [3], [4], we develop a theoretical channel model for UG2UG links at the $300-900 \mathrm{MHz}$ range and empirical evaluations of UG2UG communication are reported in [10].

In addition to theoretical studies, very few WUSN experiments have been performed to date. Experiment results in [12] show that a communication range of $7 \mathrm{~m}$ is achieved at a $6 \mathrm{~cm}$ burial depth with MicaZ motes [17]. UG2AG and AG2UG experiments using customized sensor nodes are also realized in [8], where a maximum achieved communication range of $62 \mathrm{~m}$ is reported at a burial depth of $2-4 \mathrm{~cm}$. While these results report feasibility of WUSN applications, characteristics of UG2AG and AG2UG links have not been investigated.

In [13], an ultra wideband elliptical antenna is proposed for the underground communication. The results show an adequate radiation efficiency $(>90 \%)$ of this antenna in different soil textures and moisture levels. A model exclusively for UG2AG communication is proposed in [14] and experimental results are provided using the ultra wideband elliptical antenna in [13] and a high-gain antenna for an aboveground receiver. Communication ranges of 30 and $150 \mathrm{~m}$ are reported for the burial depths of $40 \mathrm{~cm}$ and $25 \mathrm{~cm}$, respectively. However, only long range $(>20 \mathrm{~m})$ UG2AG communication links are considered.

Despite the potential applications of the existing work, a complete characterization of both UG2AG and AG2UG communication has not been performed yet. However, UG2AG and AG2UG links are essential components of a complete multi-hop network solution for WUSNs. Moreover, the experiments in [12], [8] consider only burial depths at the topsoil region $(0-30 \mathrm{~cm})$. This region is not feasible for agricultural applications, where plowing and similar mechanical activities occur at the topsoil region. Therefore, characterization of the UG2AG/AG2UG links is required at the subsoil region (30$100 \mathrm{~cm}$ ) for cost-effective solutions. In this work, we provide a characterization of the UG2AG/AG2UG communication based on experiments realized at both the subsoil and topsoil regions. More specifically, the effects of the antenna design, practical burial depths, and soil moisture on both UG2AG and AG2UG communication performance are highlighted.

\section{MATERIAls AND Methodology}

In this section, the details of the outdoor environment, hardware, software, and the methodology for the experiments are presented. The underground experiments with $433 \mathrm{MHz}$ Mica2 [17] sensor nodes were carried out in South Central Agricultural Laboratory (SCAL) of the University of Nebraska-Lincoln, located at Clay Center, NE. The analysis of the soil texture, the particle density, and the bulk density of the site is shown in Table I [18]. To observe the effects of soil moisture, two different soil moisture, i.e., volumetric water content (VWC), values are considered. Experiments realized in dry and wet conditions correspond to VWC of $9.5 \%$ and $37.3 \%$, respectively. If not explicitly stated, an experiment is realized with $\mathrm{VWC}_{d r y}$.

TABLE I

SOIL PARAMETERS USED IN THE EXPERIMENTS.

\begin{tabular}{|c|c|c|c|c|}
\hline Sample Depth & Texture & \%Sand & \%Silt & \% Clay \\
\hline $0-20 \mathrm{~cm}$ & Silt Loam & 17 & 55 & 28 \\
\hline $20-60 \mathrm{~cm}$ & Silt Clay Loam & 16 & 46 & 38 \\
\hline Particle density & Bulk density & $\mathbf{V W C}_{d r y}$ & $\mathbf{V W C}_{\text {wet }}$ & \\
\hline $2.66 \mathrm{~g} / \mathrm{cm}^{3}$ & $1.3 \mathrm{~g} / \mathrm{cm}^{3}$ & $9.5 \%$ & $37.3 \%$ & \\
\hline
\end{tabular}

The terminology used in this work is shown in Fig.1. The symbol $d_{b g}$ denotes the burial depth, which is defined as the distance between the top of the antenna of the buried node and the soil surface. The symbol $d_{a g}$ denotes the height of the aboveground node, which is defined as the distance between the center of the aboveground antenna and the surface of soil. Finally, the parameter $d_{h}$ is the horizontal internode distance between the sender and the receiver nodes. In Section III-A, the antenna schemes used in the experiments are depicted. Also, details of the construction of an ultra wideband antenna for underground operation at $433 \mathrm{MHz}$ are presented. In Section III-B, the methodology used for the experiments is explained.

\section{A. Antenna Schemes}

In the experiments, two different antenna schemes are used as follows:

Original: refers to the use of the original antenna of the $433 \mathrm{MHz}$ Mica 2 motes. This $17 \mathrm{~cm}-$ length antenna is a standard one-quarter wavelength monopole antenna. The antennas are vertically oriented according to guidelines provided in [9], [10].

FW/SEA: Full-Wave antenna combined with Single Ended Elliptical Antenna (FW/SEA) is a scheme composed of 2 
(a)

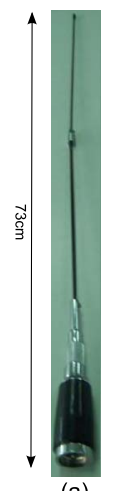

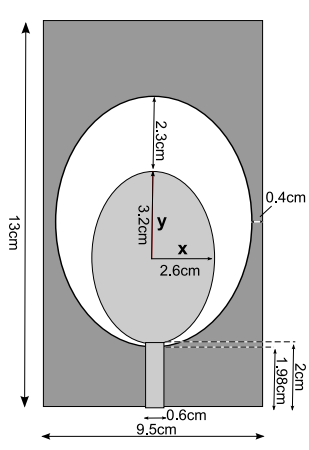

(b)
Fig. 2. (a) Commercial full-wave (FW) $433 \mathrm{MHz}$ magnetic antenna used in the aboveground sensor node. (b) Customized ultra wideband antenna (SEA) used in the buried sensor node.

different antennas. A commercial magnetic $433 \mathrm{MHz}$, fullwave $(\mathrm{FW})$, 3dBi-gain antenna is used in the aboveground node (Fig.2(a)). A customized ultra wideband single ended elliptical antenna (SEA) [6] is used in the underground node (Fig.2(b)).

The high relative permittivity of the soil causes a significant reduction of the wavelength of the EM waves. Moreover, the soil moisture is an important factor in this reduction. These facts have a significant impact on the design of an underground antenna. As an example, an antenna designed to operate at $433 \mathrm{MHz}$ in free space does not have an adequate underground performance at the same frequency because the corresponding wavelength of the signal in soil is different than in air. In other words, a buried antenna must be designed for a nominal frequency higher than the original frequency used by an aboveground antenna. More specifically, the change in wavelength of the underground signal, $\lambda$, when it propagates through the soil, compared to the wavelength, $\lambda_{0}$, in free space is given by [11]:

$$
\lambda=\frac{2 \pi}{\beta}, \beta=\frac{2 \pi c}{\lambda_{0}} \sqrt{\frac{\mu_{r} \mu_{0} \epsilon^{\prime} \epsilon_{0}}{2}\left[\sqrt{1+\left(\frac{\epsilon^{\prime \prime}}{\epsilon^{\prime}}\right)^{2}}+1\right.},
$$

where $\beta$ is the phase shifting constant in $\mathrm{radian} / m, \lambda$ is the wavelength of the underground signal, $\lambda_{0}$ is the wavelength of the signal in free space, $\mu_{r}$ is the relative permeability of the soil ( $\mu_{r}=1$ for non-magnetic soil), $\mu_{0}=4 \pi * 10^{-7} \mathrm{~N} / A^{2}$ is the magnetic constant, and $\epsilon^{\prime}$ and $\epsilon^{\prime \prime}$ are the real and imaginary parts, respectively, of the relative permittivity of the soil.

The dielectric properties of the soil, given by the parameters $\epsilon^{\prime}$ and $\epsilon^{\prime \prime}$ are significantly affected by the changes in soil properties as modeled by the Peplinski semi-empirical dielectric mixing model for the $0.3-1.3 \mathrm{GHz}$ band [5]. The soil parameters shown in Table I and the operating frequency of $433 \mathrm{MHz}$ are used as input values for the Peplinky's model. The minimum and maximum measured VWC values observed in the experiments site are also used as boundary conditions. The numerical results show that the antenna for this environment needs to have a dynamic wavelength ranging from 30 to $69 \mathrm{~cm}$. These values correspond to antennas that work on the 1 to $1.8 \mathrm{GHz}$ range in free space. Accordingly, an ultra wideband (UWB) antenna is used in the experiments, which has good performance for a wide range of frequencies based on the Single Ended Elliptical Antenna (SEA) for 3.1 - 10.6 $\mathrm{GHz}$ proposed in [6]. As a result, a customized SEA for underground operation at $433 \mathrm{MHz}$ is developed considering a theoretical lower end frequency of $1 \mathrm{GHz}$. The physical measurements of the SEA are shown in Fig.2(b).

\section{B. Software and Methodology}

For the experiments, a Java/TinyOS 1.1x application, called S-GriT (Small Grid Testbed for WSN Experiments) [9], [10], is developed to enable carrying out several experiments without reprogramming the sensor nodes and without the use of cables connecting the sender-receiver pair of nodes. The S-GriT allows configuration of multiple experiments with the following parameters: transmit power level, number of messages for the experiment, number of bytes per message, and delay between the transmission of each message. For the experiments, the packet error rate (PER) and the RSS level for the received packet are collected. The maximum transmit power level of the Mica2 $(+10 \mathrm{dBm})$ is used. The size of each packet is 37 bytes and a $100 \mathrm{~ms}$ delay between each packet transmission is configured. Each experiment is based on a set of 3 tests with 350 messages each, which result in a total of 1050 packets. The number of packets correctly received by the receiver node is recorded along with the received signal strength (RSS) for each packet.

\section{EXPERIMENT RESULTS}

In this section, an empirical analysis of the impacts of antenna design, burial depth, and soil moisture on UG2AG and AG2UG communication performance is presented.

\section{A. Effects of the Antenna Design}

To illustrate the effects of antenna design on the UG2AG and AG2UG communication performance, experiments are performed with the original and the FW/SEA antenna schemes, where the depth $\left(d_{b g}=35 \mathrm{~cm}\right)$ and the height $\left(d_{a g}=2.5 \mathrm{~m}\right)$ are fixed. The horizontal inter-node distance, $d_{h}$, is varied from 0 to $25 \mathrm{~m}$. In Figs.3(a) and 3(b), the RSS and PER values are shown, respectively, as a function of the horizontal inter-node distance, $d_{h}$. Each line in the figures shows the results for both antenna schemes and for UG2AG and AG2UG links.

As shown in Fig.3(a), an increase in the horizontal internode distance, $d_{h}$, decreases the signal strength, as expected. However, in the region close to the buried sensor $\left(d_{h}<1 \mathrm{~m}\right)$, the signal strength is not maximum. This is related to the nearfield effects of the omnidirectional antennas. It is well known that such antennas present nulls, or regions, where the signal is strongly attenuated in the locations very close to the antenna axis, i.e., on top of the underground antenna. As shown in Figs. 3(a) and 3(b), the FW/SEA scheme combats the adverse effects of the change in wavelength in soil and clearly enhances both UG2AG and AG2UG communication performance. For instance, using the original antenna scheme, no AG2UG communication is possible and the $\mathrm{UG} 2 \mathrm{AG}$ communication range is $6 \mathrm{~m}$. On the other hand, the FW/SEA scheme improves the $\mathrm{UG} 2 \mathrm{AG}$ and $\mathrm{AG} 2 \mathrm{UG}$ communication ranges to approximately 21 and $11 \mathrm{~m}$, respectively. It is also possible to observe an 


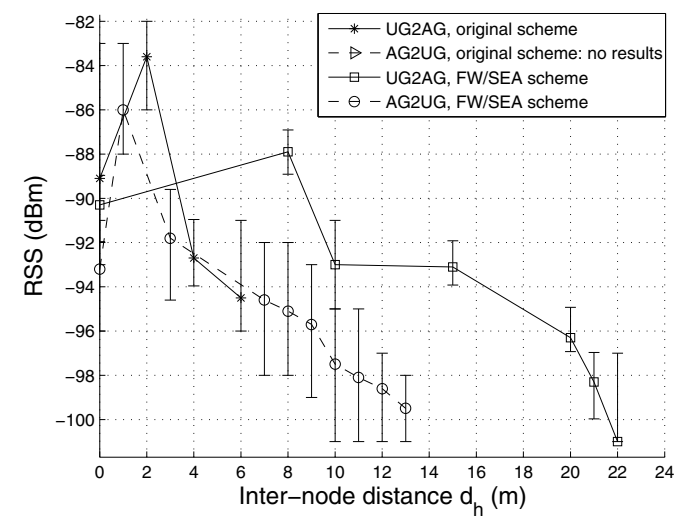

(a)

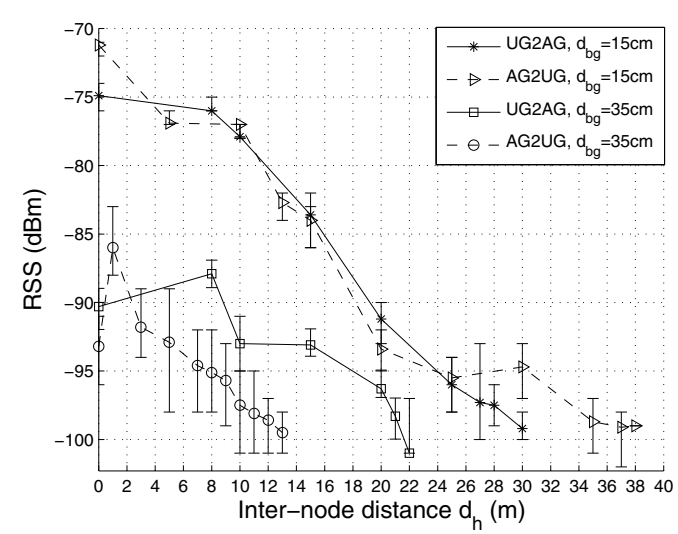

(c)

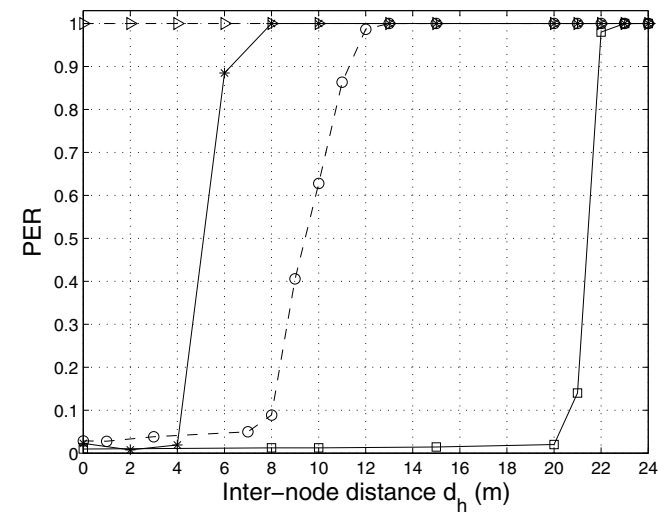

(b)

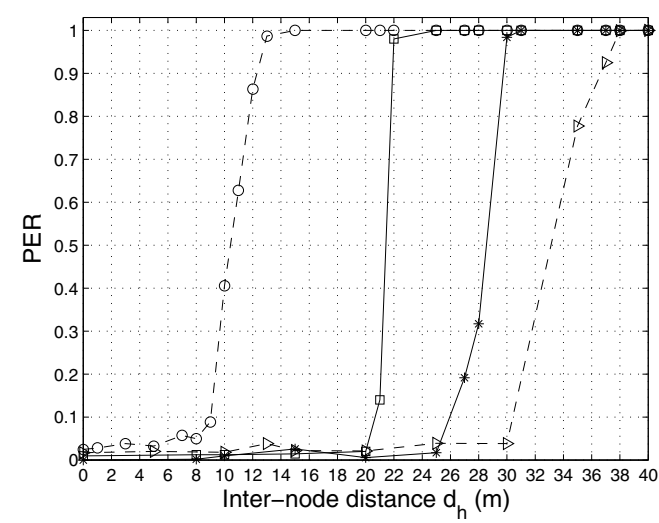

(d)

Fig. 3. Effects of the antenna scheme and burial depth on the UG2AG and AG2UG communication performance. (a) RSS and (b) PER for the different antenna schemes: original antennas and FW/SEA. (c) RSS and (d) PER for different burial depths: $15 \mathrm{~cm}$ and $35 \mathrm{~cm}$ and with the FW/SEA scheme.

asymmetry between UG2AG and AG2UG links in Figs. 3 which is discussed in Section IV-B.

\section{B. Effects of Burial Depth}

To investigate the effects of the burial depth on the signal strength and PER, the FW/SEA antenna scheme is used with a fixed height, $d_{a g}$, of $2.5 \mathrm{~m}$, burial depths, $d_{b g}$, of 15 and $35 \mathrm{~cm}$, and the horizontal inter-node distance, $d_{h}$, is varied from 0 to 40m. In Figs. 3(c) and 3(d), the RSS and PER values are shown, respectively, as a function of the horizontal inter-node distance.

As shown in Fig.3(c), an increase in the horizontal internode distance, $d_{h}$, decreases the signal strength, as expected. For both UG2AG and AG2UG communication, the higher burial depth $\left(d_{b g}=35 \mathrm{~cm}\right)$ is associated with an increase in attenuation. For instance, at $d_{b g}=15 \mathrm{~cm}$ and $d_{h}=8 \mathrm{~m}$, the signal strength for both $\mathrm{UG} 2 \mathrm{AG}$ and $\mathrm{AG} 2 \mathrm{UG}$ is $-76 \mathrm{dBm}$. However, for a deeper installation $\left(d_{b g}=35 \mathrm{~cm}\right)$ and the same internode distance, the received signal strength are $-88 \mathrm{dBm}$ and $-95 \mathrm{dBm}$ for UG2AG and AG2UG, respectively. Since a higher burial depth also implies an increase in the soil path, higher attenuation is observed at higher depth [10]. It can also be observed in Fig.3(c) that the variance of RSS is slightly larger for the $\mathrm{AG} 2 \mathrm{UG}$ communication at $d_{b g}=35 \mathrm{~cm}$. This result suggests that the multi-path effects are particularly stronger for the combination of AG2UG and high burial depths. It is well known that EM waves that propagate from a medium with a lower dielectric constant (air) to other one with a higher constant (soil) are highly reflected. Consequently, the air-soil interface causes an additional attenuation for the AG2UG communication.

As shown in Figs. 3(c) and 3(d), the shallower burial depth of the node can significantly enhance both UG2AG and AG2UG communication. For instance, when the burial depth changes from $35 \mathrm{~cm}$ to $15 \mathrm{~cm}$, a decrease of $9 \mathrm{~dB}$ and $16 \mathrm{~dB}$ on the average attenuation are observed for the UG2AG and AG2UG links, respectively. As shown in Fig. 3(d), the communication range is extended by approximately $40 \%$ and $300 \%$ for UG2AG and AG2UG, respectively. The significant increase in the communication range of AG2UG link reveals that the AG2UG link has a higher sensitivity with respect to the soil properties. The impacts of this higher sensitivity are shown in Fig.3(d). It can be observed that the transitional region [16], where the PER significantly varies, is very small for UG2AG links. For instance, at $d_{b g}=35 \mathrm{~cm}$, a high quality (PER $<10 \%)$ UG2AG communication is possible with an inter-node distance of up to $20 \mathrm{~m}$ and the communication is completely interrupted at a distance of $22 \mathrm{~m}$ (a $2 \mathrm{~m}$ increase). On the other hand, for the AG2UG link, at the same burial depth, the transitional region shown in Fig. 3(d) is significantly 


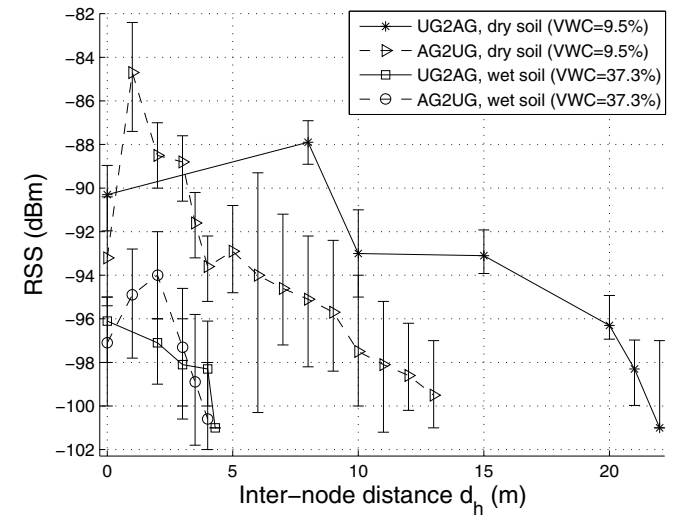

(a)

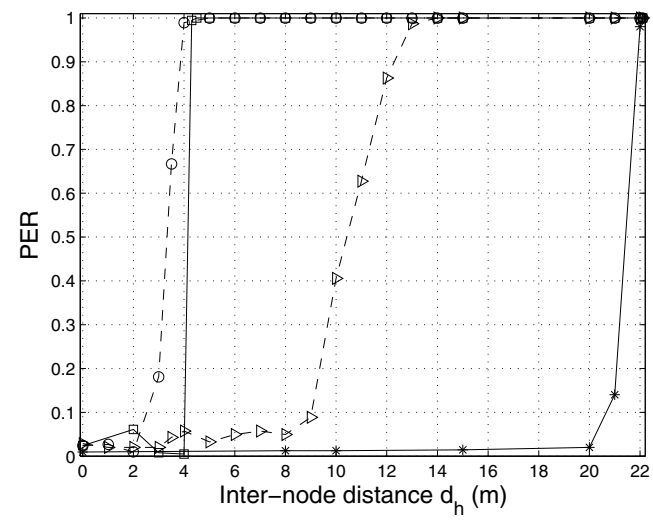

(b)

Fig. 4. Effects of the soil moisture on the UG2AG and AG2UG communication.

larger, and ranges between $d_{h}=9 \mathrm{~m}$ to $14 \mathrm{~m}$.

UG2AG and AG2UG links exhibit asymmetry with respect to the maximum communication range at each direction. Let us define symmetric region as the range of inter-node distances where the PER of the UG2AG link does not differ by more than $10 \%$ of the PER of the AG2UG link. In this region, reliable communication between an underground and an aboveground node can be performed due to the existence of bi-directional communication links. Denoting the maximum distance for the symmetric region as $d_{\text {sym }}$, it can be observed from Fig.3(d) that for burial depths $d_{b g}=15 \mathrm{~cm}$ and $d_{b g}=35 \mathrm{~cm}$, $d_{\text {sym }}=25 \mathrm{~m}$ and $d_{\text {sym }}=9 \mathrm{~m}$, respectively. In the remaining regions of the communication range, only the UG2AG link or the AG2UG link exists. For instance, for $d_{b g}=35 \mathrm{~cm}, \mathrm{UG} 2 \mathrm{AG}$ link has a longer range compared to the AG2UG link, whereas for $d_{b g}=15 \mathrm{~cm}$, AG2UG link has a better performance.

These results highlight the asymmetry between the UG2AG and AG2UG links and reveal that either direction can dominate communication success based on the burial depth. Four factors contribute for the overall signal attenuation: (a) the air path loss, (b) the soil-air/air-soil interface, (c) the multi-path effects from the soil surface, and (d) the soil path loss. The air and soil path losses are basically the same for both UG2AG and AG2UG links. Therefore, the observed asymmetry are directly associated with the factors (b) and (c). For instance, in Fig.3(c), considering the AG2UG link and $d_{b g}=15 \mathrm{~cm}$, it is observed that the RSS is almost the same for the region $20<d_{h}<30 \mathrm{~m}$. Therefore, the factors (b) and (c) change with the location and cancel the additional attenuation due to the increasing air path loss. The Snell's Law supports the dependency of the factors (b) and (c) on the distances related to the nodes $\left(d_{h}, d_{a g}, d_{b g}\right)$ and on the direction of the link (UG2AG or AG2UG). Moreover, the different radiation patterns of the antennas (FW and SEA) contribute for the non-monotonic asymmetry. From the results, the most important aspect related to this asymmetry is that shallower burial depth, i.e. $15 \mathrm{~cm}$, results in lower attenuation for the air-soil interface, which significantly enhances the AG2UG link performance.

\section{Effects of Soil Moisture}

In this section, the effects of soil moisture on the signal strength and PER are discussed. In the experiments, the depth $\left(d_{b g}=35 \mathrm{~cm}\right)$ and the height $\left(d_{a g}=2.5 \mathrm{~m}\right)$ are fixed and the horizontal inter-node distance, $d_{h}$, is varied from 0 to $22 \mathrm{~m}$ for two soil moisture levels, $V W C_{d r y}(9.5 \%)$ and $V W C_{w e t}$ $(37.3 \%)$. The latter VWC value represents the saturation point of the soil in our testbed. In Figs. 4(a) and 4(b), the RSS and PER values are shown, respectively, as a function of the horizontal inter-node distance.

As shown in Fig.4(a), the variation from $9.5 \%$ to $37.3 \%$ in the VWC level causes an additional attenuation of $8 \mathrm{~dB}$, on the average, for both UG2AG and AG2UG. Consequently, the communication range decreases by approximately $80 \%$ and $70 \%$ for $\mathrm{UG} 2 \mathrm{AG}$ and $\mathrm{AG} 2 \mathrm{UG}$ links, respectively. An increase in the soil moisture has two main effects on the wireless underground communication. First, the wavelength of the signal is decreased, which causes indirect loss due to the antenna mismatch, as explained in Section III. The FW/SEA scheme reduces this first effect. Second, an increase in soil moisture significantly increases the soil attenuation [5], [4], [10]. This result shows that soil moisture has a significant influence on the quality of UG2AG and AG2UG communication. Therefore, the design of the WUSN protocols should carefully adapt to the variation of the soil moisture.

\section{CONCLUSIONS}

Despite their potential, the proliferation of WUSNs has been delayed by the unique challenges of the underground environment. Some of these challenges are related to the communication involving an underground node and an aboveground device. Accordingly, this work highlights the characteristics of the UG2AG and AG2UG communication based on empirical results. More specifically, the effects of the antenna design, burial depth, and soil moisture on both UG2AG and AG2UG communication performance are investigated. An overview of the experiment results are shown in Table II. In this table, the normalized transitional region width, $\Gamma$, is given by [16]:

$$
\Gamma=\frac{d_{e}^{t r}-d_{b}^{t r}}{d_{b}^{t r}},
$$


TABLE II

OVERVIEW OF RESULTS

\begin{tabular}{|c|c|c||c|c|c|c|}
\hline $\begin{array}{c}\text { Comm. } \\
\text { Type }\end{array}$ & $\begin{array}{c}\text { Antenna } \\
\text { Scheme }\end{array}$ & $\begin{array}{c}\text { Soil } \\
\text { Moisture }\end{array}$ & $\begin{array}{c}\text { Burial } \\
\text { Depth }\end{array}$ & $\begin{array}{c}\text { Maximum } \\
\text { Range }\left(d_{b}^{t r}\right)\end{array}$ & $\begin{array}{c}\text { Transitional Region } \\
\text { Coefficient }(\Gamma)\end{array}$ & $\begin{array}{c}\text { Average } \\
\text { Variance }\end{array}$ \\
\hline \multirow{4}{*}{$U G 2 A G G$} & original & Dry & $15 \mathrm{~cm}$ & $11 \mathrm{~m}$ & 0.18 & 2.2 \\
& FW/SEA & Dry & $15 \mathrm{~cm}$ & $30 \mathrm{~m}$ & 0.16 & 3.0 \\
& original & Dry & $35 \mathrm{~cm}$ & $6 \mathrm{~m}$ & 0.33 & 5.2 \\
& FW/SEA & Dry & $35 \mathrm{~cm}$ & $22 \mathrm{~m}$ & 0.04 & 2.8 \\
& FW/SEA & Wet & $35 \mathrm{~cm}$ & $4.3 \mathrm{~m}$ & 0.06 & 3.5 \\
\hline \multirow{4}{*}{$A G 2 U G$} & FW/SEA & Dry & $15 \mathrm{~cm}$ & $38 \mathrm{~m}$ & 0.21 & 2.4 \\
& original & Dry & $35 \mathrm{~cm}$ & no comm. & - & - \\
& FW/SEA & Dry & $35 \mathrm{~cm}$ & $13 \mathrm{~m}$ & 0.30 & - \\
& original & Wet & $35 \mathrm{~cm}$ & no comm. & - & 5.0 \\
& FW/SEA & Wet & $35 \mathrm{~cm}$ & $4 \mathrm{~m}$ & 0.25 & \\
\hline
\end{tabular}

where $d_{b}^{t r}$ and $d_{e}^{t r}$ are the beginning and end of the transitional region, respectively. The link presents PER $>10 \%$ for internode distances higher than $d_{b}^{t r}$. No communication is observed for distances higher than $d_{e}^{t r}$.

We have shown that the antenna design is a key aspect in the WUSN design because of the adverse effects of soil and moisture on the wavelength of the EM waves that propagate through soil. The FW/SEA antenna scheme increases the communication range by more than $350 \%$ compared to the original antennas. An ultra wideband antenna proved to be a good choice for the underground node, as indicated in [6]. The results also suggest that even better communication performance can be achieved if the antenna scheme is specifically tailored to the deployment aspects of the final WUSN application, such as the burial depth.

The strong effect of the burial depth on the communication performance, especially for the AG2UG, is also an important aspect. When the burial depth changes from $35 \mathrm{~cm}$ to $15 \mathrm{~cm}$, the communication range is extended by almost $40 \%$ and $300 \%$ for UG2AG and AG2UG links, respectively. Moreover, the burial depth also affects the asymmetry between UG2AG and AG2UG communication. For a $15 \mathrm{~cm}$ burial depth, AG2UG communication has a higher communication range than UG2AG. On the other hand, for a $35 \mathrm{~cm}$ burial depth, the UG2AG communication has better performance. This strong dependence on soil properties motivates the development of environment-aware channel models for WUSNs, which constitutes our future work.

The non-monotonic asymmetric behavior of UG2AG and AG2UG links has a strong impact on the design and development of multi-hop networking protocols for WUSNs. For each pair of underground and aboveground nodes, there is a symmetric region, where UG2AG and AG2UG links present similar performance. This symmetric region enables the development of reliable communication schemes between a WUSN and aboveground devices.

Finally, the results reveal that a $21 \%$ increase in the soil moisture decreases the communication range by more than $70 \%$. These results have a significant impact on the development of multi-hop networking protocols for WUSNs and agree with the results for UG2UG communication [10].

\section{ACKNOWLEDGMENT}

This work is partly supported by the UNL Research Council Maude Hammond Fling Faculty Research Fellowship, UNL
Water Center, and USGS. The authors would like to thank Dr. Suat Irmak for his valuable comments throughout the development of the experiments and William Rathje for his continuous support during the experiments at Clay Center.

\section{REFERENCES}

[1] I. F. Akyildiz, W. Su, Y. Sankarasubramaniam, E. Cayirci, "Wireless sensor networks: a survey," Computer Networks Journal (Elsevier), vol. 38, no. 4, pp. 393-422, March 2002.

[2] I. F. Akyildiz and E. P. Stuntebeck, "Wireless underground sensor networks: Research challenges," Ad Hoc Networks Journal (Elsevier), vol. 4, pp. 669-686, July 2006.

[3] I. F. Akyildiz, Z. Sun, and M. C. Vuran "Signal Propagation Techniques for Wireless Underground Communication Networks," Physical Communication Journal (Elsevier), vol. 2, no. 3, pp. 167-183, Sept. 2009.

[4] L. Li, M. C. Vuran, and I. F. Akyildiz, "Characteristics of Underground Channel for Wireless Underground Sensor Networks," in Proc. Med-HocNet '07, Corfu, Greece, June 2007.

[5] N. Peplinski, F. Ulaby, and M. Dobson, "Dielectric properties of soils in the 0.3-1.3-GHz range," IEEE Trans. Geoscience and Remote Sensing, vol. 33, no. 3, pp. 803-807, May 1995.

[6] J. Powell and A. Chandrakasan, "Differential and Single Ended Elliptical Antennas for 3.1-10.6 GHz Ultra Wideband Communication," in Antennas and Propagation Society International Symposium, vol. 2, pp. 2935-2938, June 2004.

[7] T. S. Rappaport, "Wireless Communications - principles \& Practice," First Edition, Prentice Hall PTR, 1996.

[8] C. Ritsema et al. "A new wireless underground network system for continuous monitoring of soil water contents," Water Resources Research Journal, Vol.45, pp.1-9, May 2009.

[9] A. R. Silva and M. C. Vuran, "Development of a Testbed for Wireless Underground Sensor Networks," to appear in EURASIP Journal on Wireless Communications and Networking, special issue on "Simulators and Experimental Testbeds Designs and Development for Wireless Networks", 2010.

[10] A. R. Silva and M. C. Vuran, "Empirical Evaluation of Wireless Underground-to-Underground Communication in Wireless Underground Sensor Networks," in Proc. IEEE DCOSS '09, Marina Del Rey, CA, June 2009.

[11] J. A. Stratton, "Electromagnetic Theory," Wiley-IEEE Press , 2007.

[12] E. Stuntebeck, D. Pompili, T. Melodia, "Underground Wireless Sensor Networks Using Commodity Terrestrial Motes," poster presentation at IEEE SECON 2006, September 2006.

[13] M. J. Tiusanen, "Wideband Antenna for Underground Soil Scout Transmission," IEEE Antennas and Wireless Propagation Letters, Vol.5, No.1, pp.517-519, December 2006.

[14] M. J. Tiusanen, "Wireless Soil Scout prototype radio signal reception compared to the attenuation model," Precision Agriculture Journal, vol. 10, no. 5, pp. 372-381, Oct. 2009.

[15] M. C. Vuran and I. F. Akyildiz, "Packet Size Optimization for Wireless Terrestrial, Underwater, and Underground Sensor Networks," in Proc. IEEE INFOCOM '08, Phoeniz, AZ, April 2008.

[16] M. Zuniga and B. Krishnamachari, "An analysis of unreliability and asymmetry in low-power wireless links," in ACM Transactions on Sensor Networks, Vol.3, No.2, June 2007.

[17] Crossbow MICA2 and MICAz motes, http://www.xbow.com.

[18] Ward Laboratories, http://wardlab.com. 